\title{
Ballistic Transport Phenomena in Nanostructures of Paraelectric Lead Telluride
}

\author{
G. GRABECKI \\ Institute of Physics, Polish Academy of Sciences \\ al. Lotników 32/46, 02-668 Warszawa, Poland
}

This is a review of our recent developments in the physics of lead telluride nanostructures. PbTe is a IV-VI narrow gap paraelectric semiconductor, characterized by the huge static dielectric constant $\varepsilon>1000$ at helium temperatures. We nanostructurized this material by means of e-beam lithography and wet chemical etching of modulation doped $\mathrm{PbTe} / \mathrm{Pb}_{1-x} \mathrm{Eu}_{x} \mathrm{Te}$ quantum wells. Magnetoresistance measurements performed on the nanostructures revealed a number of magnetosize effects, confirming a ballistic motion of the carriers. The most important observation is that the conductance of narrow constrictions shows a precise zero-field quantization in $2 e^{2} / h$ units, despite a significant amount of charged defects in the vicinity of the conducting channel. This unusual result is a consequence of a strong suppression of the Coulomb potential fluctuations in $\mathrm{PbTe}$, an effect confirmed by numerical simulations. Furthermore, the orbital degeneracy of electron waveguide modes can be controlled by the width of $\mathrm{PbTe} / \mathrm{Pb}_{1-x} \mathrm{Eu}_{x}$ Te quantum wells, so that unusual sequences of plateau conductance can be observed. Finally, conductance measurements in a nonlinear regime allowed for an estimation of the energy spacing between the one-dimensional subbands.

PACS numbers: 72.80.Jc, 73.21.Hb, 73.23.Ad, 77.22.Ch

\section{Introduction}

Recently, one has observed a great interest in quantum nanostructures hosting controllable quantum states necessary for the hardware basis of quantum information and communication technologies $[1,2]$. Canonical examples of such devices are one-dimensional (1D) quantum wires (QWRs), whose conductance is quantized in $2 e^{2} / h$ units [3], as well as quantum dots revealing discrete energy levels, in a close analogy to atomic levels [4]. However, the realization of large matrices of such devices necessary for building the quantum computers requires a high degree of reproducibility. This is not easy because of the intrinsic parameter fluctuations 
introduced by discreteness of charge and matter, appearing when the device size becomes scaled to nanometer dimensions [5]. In the case of semiconductor nanostructures, the discrete nature of doping (necessary for providing the free carriers) comes to the view. Since the side effect of doping is the occurrence of ionized impurities, their Coulomb potentials produce random fields locally distorting the potential landscape within the nanostructures. Such spurious fluctuations cannot be screened out due to a very weak screening in low-dimensional systems and a low number of mobile charges. Modulation doping, in which the doped region is spatially separated from the free carriers, has been utilized as a mean to alleviate the problem, however, even in this case one still may observe strong effects caused by fluctuations produced by the long-range tails of the Coulomb potentials [6].

Although the above problem seems to be fundamental and not easily solvable, we show that it can be, at least, partially reduced in nanostructures of a medium of a very large dielectric constant. In such a case the Coulomb potentials are strongly suppressed and, thus, the role of individual charged impurities is reduced. Consequently, the random potential fluctuations may be reduced much below the separation of the quantum energy levels in the nanostructures. We will show that all this is possible in lead telluride, PbTe. This compound of IV-VI group is a narrow gap semiconductor with the rock-salt structure. It has a multivalley band structure with anisotropic valleys residing in the $L$ of the Brillouin zone. It possesses excellent semiconducting properties [7], namely a high carrier mobility up to $2 \times 10^{6} \mathrm{~cm}^{2} /(\mathrm{V} \mathrm{s})$, and controllability of electron or hole concentrations by either doping or annealing. However, the most important property of $\mathrm{PbTe}$ is its paraelectric character leading to a huge dielectric constant, $\varepsilon=1350$ at $4.2 \mathrm{~K}$ [8]. This stems from the fact that $\mathrm{PbTe}$ is in a proximity to a lattice instability, which leads to softening of the transverse optical phonon frequency.

It is well known that the observation of quantum ballistic effects requires extremely pure devices, with a defect concentration as low as possible. In particular, the quality of the quantization is extremely sensitive to any potential irregularities within the constriction because they lead to backscattering of the electron waves. For this reason, observations of the conductance quantization can be used as a tool for detecting potential fluctuations in the host medium. Accordingly, we have demonstrated [9-15] that in PbTe nanostructures the amplitude of potential fluctuations is strongly suppressed, even in the presence of a significant concentration of charged impurities in the close vicinity of the device. This is concluded from the pronounced 1D quantization in a modulation doped $\mathrm{PbTe}$ wires, indicating a rather small backscattering, despite only a few nm separation of the conducting channel from the heavily doped layer.

Another consequence of the huge dielectric constant is a very small Coulomb charging energy. In particular, we have not observed any indications of the Coulomb blockade in PbTe quantum dots [11]. Potentially, $\mathrm{PbTe}$ would offer the possibility of tuning the Coulomb charging energy independently of the device size. 
Such property would be very useful for realization of the quantum entanglement in the quantum dots [16].

Persistence of the quantum ballistic regime in $\mathrm{PbTe}$ nanostructures enables extensive studies of many physical phenomena. Because the electric fields are small, the quantum wells conserve their rectangular shape even in the case of modulation doping. Therefore, the 2D subband energies are exclusively determined by the well width. This allows for obtaining QWRs of different aspect ratios and different degeneracy of the transverse modes in the electron waveguides $[10,13]$. Another important property results from the narrow-gap character of $\mathrm{PbTe}$. For appropriate crystallographic orientations, there is a large magnitude of the Landé factor of electrons, $g^{*} \approx 60$. Accordingly, the quantized conductance shows a visible spin-splitting of the plateaus already in magnetic fields about $0.2 \mathrm{~T} \mathrm{[10]}$. It is well known that if the Fermi energy is adjusted to the spin-gap of the lowest 1D subband, the quantum point contact becomes a spin-dependent barrier. Therefore, it can be exploited to develop devices generating spin-polarized current at a small spatial scale [17]. Importantly, in the case of PbTe the necessary magnetic field is so low that it could be generated locally, e.g. by means of deposited micromagnets [18]. In the following sections we give a review of our works on PbTe nanostructures.

\section{Properties of initial PbTe quantum wells}

In order to nanostructurize $\mathrm{PbTe}$, we needed the material in a form of thin layers deposited in an insulating substrate. For this purpose, we have used $\mathrm{PbTe}$ quantum wells (QWs) grown by molecular beam epitaxy (MBE) onto (111) $\mathrm{BaF}_{2}$ substrates. The growth was performed at the University of Linz (Austria), according to the procedures described in [19]. The initial layer schematic cross-section is shown in Fig. 1a. The PbTe single QW resides between $\mathrm{Pb}_{0.92} \mathrm{Eu}_{0.08} \mathrm{Te}$ barriers.
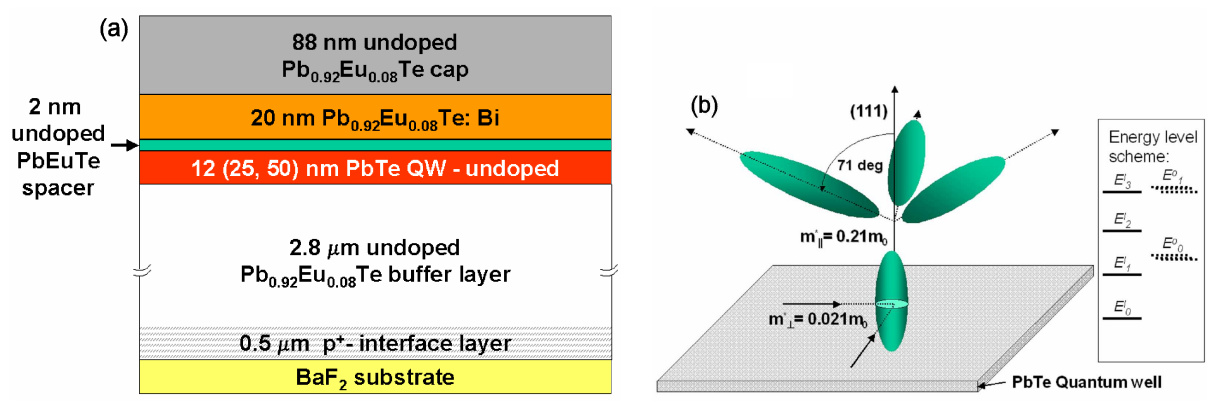

Fig. 1. (a) Schematic view of the initial multilayer used for nanostructurization of PbTe. (b) PbTe conduction band valley orientation with respect to the initial quantum well. Schematic representation of the $2 \mathrm{D}$ subband structure is shown on the right. 
For our studies, we have used 12, 25, and $50 \mathrm{~nm}$ wide QWs. For an Eu composition of $8 \%$, the energy gap is equal to $0.66 \mathrm{eV}$. Since the band-offset in this system is close to 0.5 [20], the barrier height is estimated to be about $235 \mathrm{meV}$.

It has to be stressed that the initial fourfold $L$-valley degeneracy of the $\mathrm{PbTe}$ bands is partially lifted in the QWs grown along (111) direction. This is illustrated in Fig. 1b. Since the parallel effective mass $m_{\|}^{*}$ is largest, the quantum confinement shifts down the energy of the single valley with the long axis parallel to the growth direction and it forms the ground state. It is a beginning of the ladder of the two-dimensional (2D) subbands represented by solid lines. The electrons occupying these subbands are characterized by a very small in-plane effective mass, $m_{\perp}^{*}$, and thus are expected to be very mobile. The three obliquely oriented valleys form the second ladder of 2D subbands, with the onset placed at a higher energy. In the case of unperturbed QW, they remain triply degenerated, and their intersubband spaces are larger than those of the parallel valley subbands (see dotted lines in Fig. 1b).

In order to introduce conducting electrons into the $\mathrm{QW}$ we have used the modulation doping scheme. Therefore, the front $\mathrm{Pb}_{0.92} \mathrm{Eu}_{0.08}$ Te barrier is doped with $\mathrm{Bi}\left(N_{\mathrm{D}} \approx 3 \times 10^{18} \mathrm{~cm}^{-3}\right)$. The heavily doped layer is separated from the quantum well by an undoped $2 \mathrm{~nm}$ wide spacer layer. Let us note that it is much thinner than usually applied in other systems (e.g. heterostructures of III-V materials $[3,6])$. Standard transport measurements reveal the electron densities $n_{2 \mathrm{D}}$ ranging from $1 \times 10^{12} \mathrm{~cm}^{-2}$ to $1 \times 10^{13} \mathrm{~cm}^{-2}$ and electron mobilities not exceeding $1 \times 10^{5} \mathrm{~cm}^{2} /(\mathrm{V} \mathrm{s})$ at $4.2 \mathrm{~K}$. This corresponds to the electron mean free paths $l_{\mathrm{e}} \leq 3 \mu \mathrm{m}$. In Fig. 2, we show Shubnikov-de Haas oscillations observed in

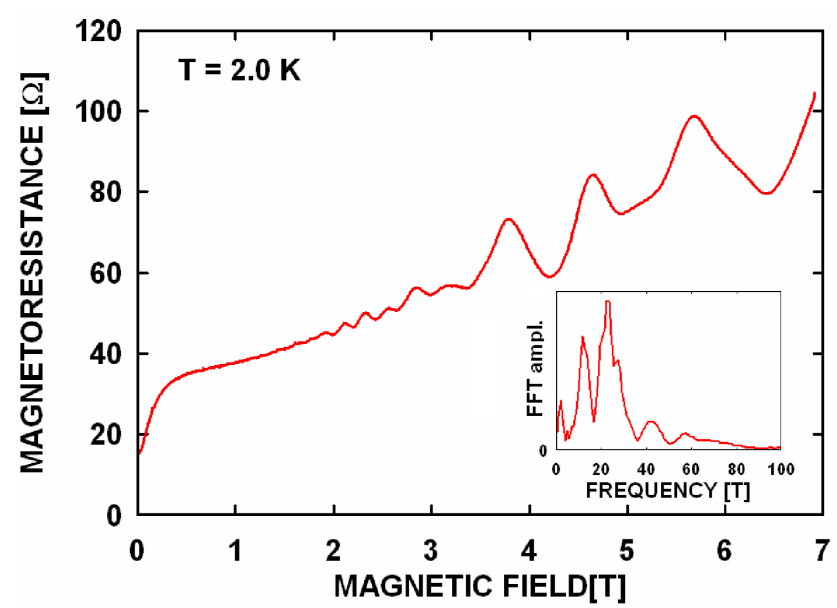

Fig. 2. Shubnikov-de Haas oscillations measured in $\mathrm{PbTe} / \mathrm{Pb}_{0.92} \mathrm{Eu}_{0.08} \mathrm{Te}$ single QW of the width $12 \mathrm{~nm}$. Inset shows the Fourier analysis of the oscillation pattern in $1 / B$ scale. 
$12 \mathrm{~nm}$ wide $\mathrm{PbTe}$ single QW. A complicated oscillation pattern is a consequence of several subbands participating in the conductance (see the Fourier analysis in Fig. 2 inset).

It should be noted that the electron mobility is strongly reduced with respect to record values for the bulk-like PbTe epilayers (up to $2 \times 10^{6} \mathrm{~cm}^{2} /(\mathrm{V} \mathrm{s})[7]$ ) despite the application of the modulation doping. The main reason is a strong alloy scattering [21] at the $\mathrm{PbTe} / \mathrm{Pb}_{0.92} \mathrm{Eu}_{0.08} \mathrm{Te}$ interfaces. Additionally, the system contains a significant number of threading dislocations formed during the initial growth on the $\mathrm{BaF}_{2}$ surface as a result of $4.2 \%$ lattice mismatch. The application of a thick buffer layer reduces the dislocation density, but it remains still significant in the QW region (at least at the level of $10^{7} \mathrm{~cm}^{-2}$ ) [22]. The dislocation density strongly grows when approaching the substrate, and as close as $0.5 \mu \mathrm{m}$ from $\mathrm{BaF}_{2}$ a thin $p^{+}$interfacial layer is formed [23]. Therefore, together with the $n$-doped $\mathrm{QW}$, a $p-n$ junction is formed. In the next section, we employ it for gating our QWRs. Furthermore, the difference in thermal expansion coefficients between the whole structure and the $\mathrm{BaF}_{2}$ substrate produces thermal strains of the order of $0.16 \%$ when the temperature is reduced down to $4.2 \mathrm{~K}$ [11]. Finally, due to the non-stoichiometric composition, the background defect density would be as high as $10^{17} \mathrm{~cm}^{-3}$. It has to be emphasized that any of the effects enlisted above would preclude the observation of conductance quantization in any standard material. However, as we show below, this is not the case for $\mathrm{PbTe}$ due to its huge dielectric constant.

\section{Fabrication of PbTe nanostructures}

In order to form $\mathrm{PbTe}$ QWR and dots, electron-beam lithography and wet chemical etching have been used [11]. In contrast to other systems, we cannot use the split gate scheme [3], therefore the structures have a form of deeply etched mesas as shown in Fig. 3a. As it was mentioned above, for tuning the conductiv-
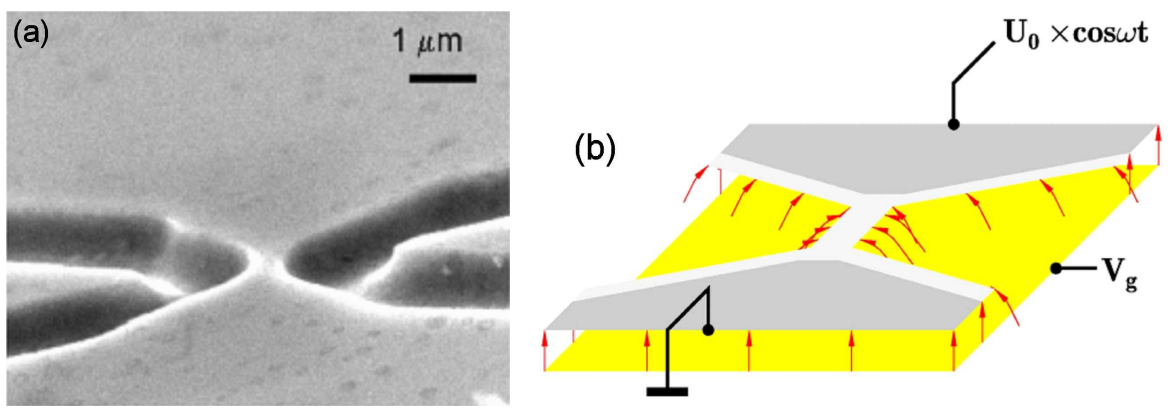

Fig. 3. (a) Scanning electron microscope image of PbTe constriction of geometrical width of about $0.5 \mu \mathrm{m}$ (from Ref. [14]). (b) Schematic representation of the $p$-type backgate operation in PbTe QWRs. 
ity in the nanostructures we employ the biasing of the naturally occurring $p-n$ junction that is formed between the $p^{+}$interfacial layer and the $n$-type quantum well [11]. The gate arrangement is schematically represented in Fig. 3b. In order to preserve electric insulation between the gate and the QW, the selective contacts for both of the $p$ - and $n$-type PbTe have been used. Because the etching depth (usually about $0.7 \mu \mathrm{m}$ ) extends only into the buffer layer and does not reach the substrate, the gate electrode extends under the entire structure. However, when negative gate voltage is applied, the narrow region is depleted prior to the pads. This is caused by a large contribution of edge effects to the capacitance and, probably, by an initial side depletion due to surface defects. In the properly prepared devices the gate resistance at $T=4.2 \mathrm{~K}$ reaches values of above $10^{9} \Omega$. The structures are very stable in time and can endure (at least) several cooling cycles between room and helium temperatures without visible worsening their properties. It should be also noted that the gate voltage $V_{\mathrm{g}}$ necessary for tuning the structures is relatively small, of the order of hundred millivolts. This is a result of high capacitance of the $p-n$ junction caused by the huge dielectric constant.

\section{Classical ballistic transport}

Classical ballistic effects can be observed if electron scattering occurs only at the device boundaries but the device size is much larger than the electron Fermi wavelength. Consequently, the conductance is determined exclusively by the nanostructure geometry. In this situation, the application of the external magnetic field leads to various magnetosize effects allowing deriving information about the

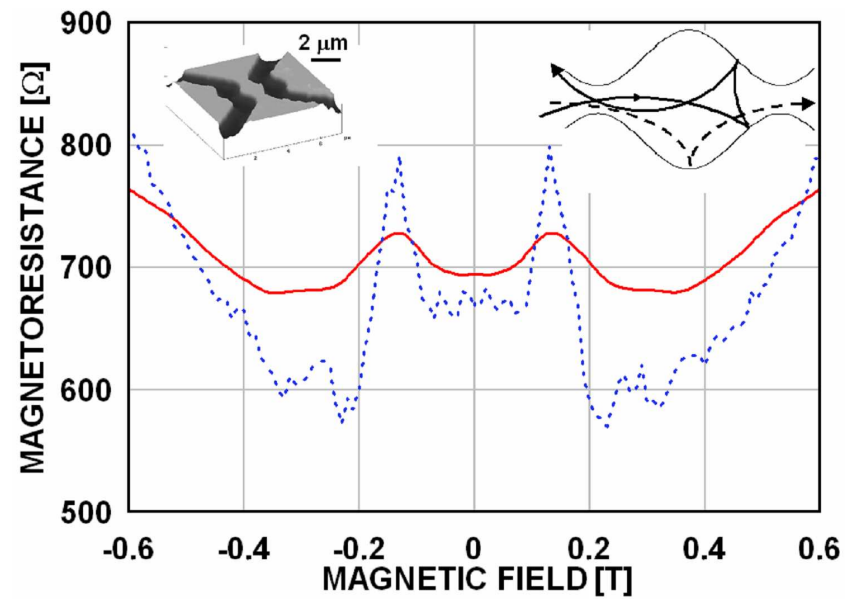

Fig. 4. Two-terminal magnetoresistance of PbTe quantum dot. Left inset represents AFM image of the actual device. Solid line - experimental data (from Ref. [11]), dotted line - numerical simulations. Right inset represents two types of characteristic electron trajectories (described in the text). 
actual device geometry [24]. A canonical example of such effects is "negative bend resistance" occurring in four-probe resistance measurements performed in a Hall cross geometry. We also have observed this phenomenon in PbTe devices of dimensions below $2 \mu \mathrm{m}[11,14]$.

In the present review, in Fig. 4, we show the magnetosize effect occurring in two-terminal magnetoresistance measured in the quantum dot geometry. The atomic force microscopy (AFM) image of the measured device is shown in the left inset. A solid curve represents experimental data. A dotted line is a result of semiclassical simulations within the electron billiard model [25], for the sample geometry shown in the right inset. As it is shown, the model reproduces well the measured magnetoresistance. In particular, there are two characteristic maxima centered around $B= \pm 0.13 \mathrm{~T}$. Around this field values, the majority of electrons entering the dot is backscattered after two subsequent reflections at the boundaries, in the way illustrated by the solid line in the right inset. Therefore, the resistance exhibits a maximum. However, if the magnetic field is increased further, the electron beam becomes guided between the entrance and the exit, as it is shown by the dotted trajectory, and this explains the subsequent resistance minimum. The observed resistance increase at still higher fields is connected with a contribution of the Hall effect in the two-probe geometry. Such good agreement of the experimental data with the billard model confirms the dominance of the ballistic transport in our devices.

\section{Quantum ballistic transport}

In order to measure the conductance quantization we have utilized narrow constrictions of geometry similar to that shown in Fig. 3a. When the temperature is lowered enough, we start to observe a sequence of quantization steps equal to $i \times\left(2 e^{2} / h\right)$, where $i$ represents integral numbers [9-11]. An example of such behavior is shown in Fig. 5, for a constriction made of $12 \mathrm{~nm}$ wide QW. The existence of such steps clearly indicates $1 \mathrm{D}$ quantization of the electron motion in the conduction channel formed by the gate voltage within the constriction. Moreover, the good quality of the steps indicates the smooth channel geometry. In fact, detailed studies of the single constriction geometry have shown that the step heights correspond to the quantized values within an accuracy of a few percent. This means that the electron transmission is close to 1, even at zero magnetic field [12]. A further evidence of weak disorder in $\mathrm{PbTe}$ constrictions has been found by conductance measurements in a millikelvin temperature range. We have not observed an occurrence of aperiodic conductance fluctuations caused by interference of electron waves due to disorder [26]. All these findings prove the efficiency of the dielectric screening of the Coulomb potentials caused by various defects existing in the vicinity of the conducting channel. This conclusion has been supported by the model calculations [12]. It is also worth noting here that we have not observed the "0.7" feature at the lowest conductance plateau [27], most probably due to the effective screening of the electron-electron interaction in $\mathrm{PbTe}$. 


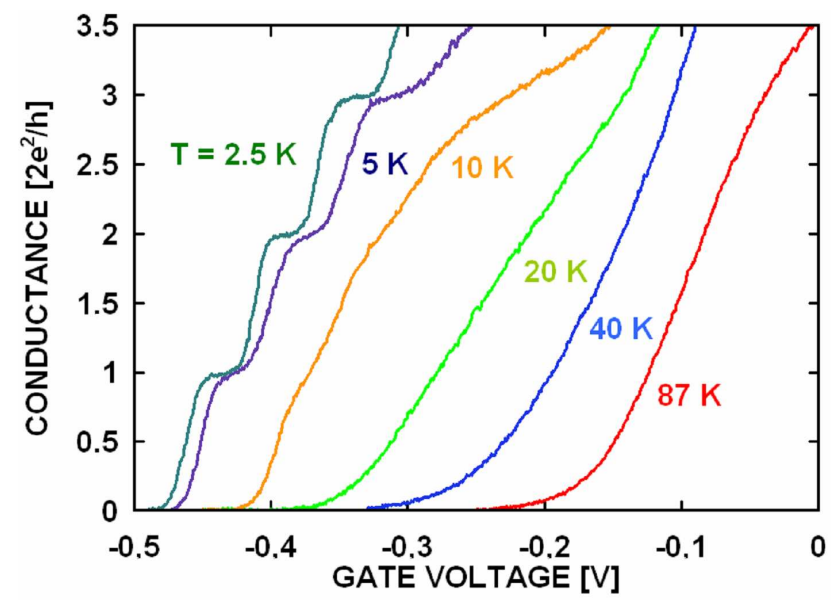

Fig. 5. Zero-field conductance measured as a function of the gate voltage in $\mathrm{PbTe}$ point contact at different temperatures.

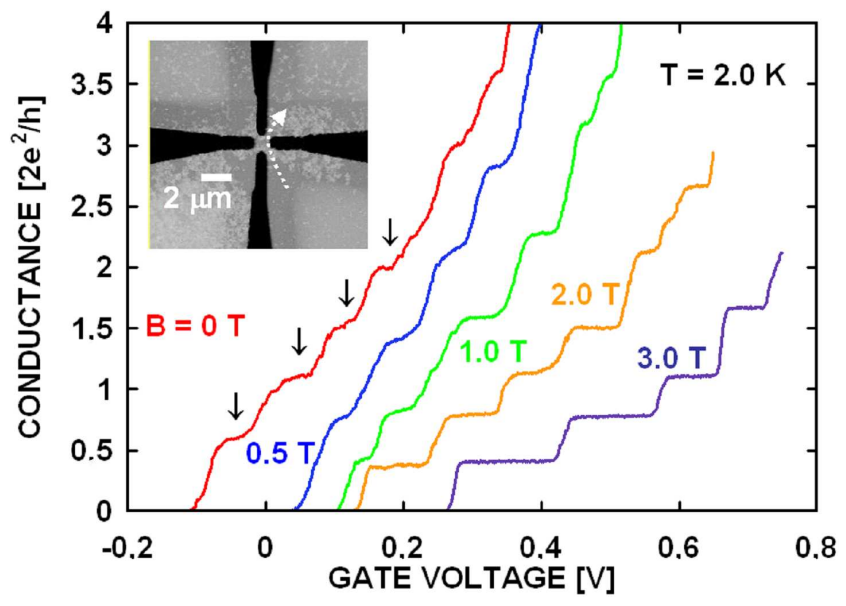

Fig. 6. Two-terminal conductance measured in PbTe Hall device represented in inset (AFM image). Arrow indicates the electric current path. Consecutive curves have been measured at different magnetic fields. They are shifted along horizontal axis to prevent overlap.

In the present review, in Fig. 6, we present supplementary data showing the conductance quantization in the Hall cross geometry. In this case, the current is passed between two adjacent arms of the device, in the way shown in the inset. Each of the arms may be regarded as a single point contact connected to the central square area. The zero-field conductance shows a sequence of steps (indicated by arrows), significantly lowered in comparison to the values of $i \times\left(2 e^{2} / h\right)$. This is a result of partial backscattering of the carriers due to the curved current path. 
When the magnetic field is raised, the backscattering becomes suppressed and the step heights are significantly increased. However, they remain lower than the expected values even at $B=3.0 \mathrm{~T}$, probably because of an addition of the serial contributions of the central area and the large contact pads. It should be also noted that for $B \geq 1 \mathrm{~T}$ we start to observe pronounced spin-resolved steps. This is a result of the large Zeeman splitting in the narrow-gap PbTe, reaching about $70 \%$ of the Landau splitting. This subject is considered in more detail in our works $[10,14]$.

\section{Orbital degeneracy of $1 \mathrm{D}$ levels in PbTe nanostructures}

In modulation doped QWs of III-V semiconductors, the total confining potential is a self-consistent result of the alloy barrier potential and the electric fields produced by the ionized impurities and the free carriers. In the case of $\mathrm{PbTe}$, the huge dielectric constant strongly reduces the electric fields making the barrier potential dominant. Therefore, the subband energies (see the scheme in Fig. 1b) are predominantly controlled by the QW width $d_{\mathrm{QW}}$. In particular, in PbTe QWRs, the initial QW width determines the cross-section shape of the electron waveguide (see the right side of Fig. 7) which in turn governs the ratio between perpendicular and lateral quantization energies [13,28]. The latter depends on the etched QWR width and may be assumed as constant. Indeed, the forms of observed conductance steps, shown in Fig. 7, result from different orbital degeneracy of
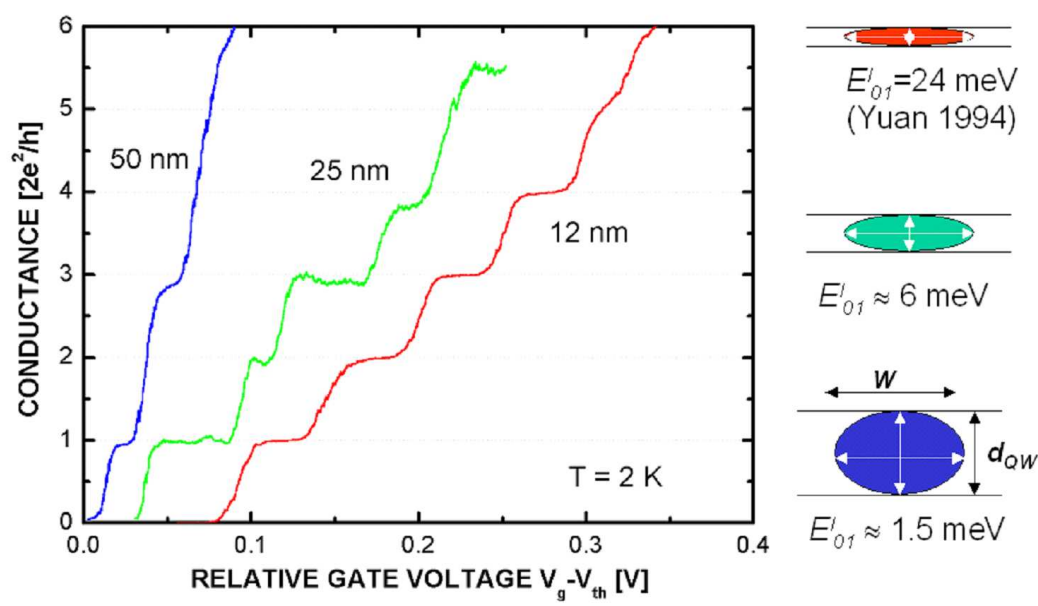

Fig. 7. Conductance (in $2 e^{2} / h$ units) as a function of the relative gate voltage $\left(V_{\mathrm{g}}-V_{\mathrm{th}}\right.$, measured from the conductance threshold) for three $\mathrm{PbTe}$ constrictions made of quantum wells of different width (indicated by numbers) (from Ref. [13]). The curves are horizontally shifted to prevent overlap. Schematic cross-sections of the constrictions are represented on the right side. 
the quantum 1D levels. The degeneracy occurs if the perpendicular and lateral quantization energies coincide. This is the case of the thickest constriction made of $50 \mathrm{~nm}$ QW (Fig. 7, leftmost curve). We observe here the step sequence $i=1$, $3,6 \ldots$ (instead of $i=1,2,3 \ldots$ ) because of orbital degeneracy of transverse modes: onefold for the ground $s$-like state, $n=1$; twofold for the first excited $p$-like state, $n=2$; threefold for the second excited $d$-like state, $n=3$, etc... For thinner QWs, the perpendicular quantization starts to dominate and the degeneracy is lifted. However, in the constriction of $25 \mathrm{~nm}$ QW, the two lowest excited 1D levels are still close one to another because the step $i=2$ is very narrow. The situation becomes most simple for the constriction of $12 \mathrm{~nm} \mathrm{QW}$, where the quantization energies are distinctly different and we observe four equal quantized steps. They correspond to four orbital non-degenerate 1D levels placed between the ground and the first excited 2D subbands in the initial QW (compare the scheme in Fig. 1b). In this range, the situation exactly corresponds to the most commonly studied QWRs of AlGaAs/GaAs heterostructures [3]. The body of the above results demonstrates clearly that $\mathrm{PbTe}$ nanostructures can be formed by imposing barriers of $\mathrm{Pb}_{1-x} \mathrm{Eu}_{x} \mathrm{Te}$.

\section{Conductance measurements in a nonlinear regime}

In the previous sections, we have considered the conductance quantization in the linear regime, for $e V_{\text {sd }} \ll \Delta E_{i j}$, where $V_{\text {sd }}$ is the source-drain voltage applied
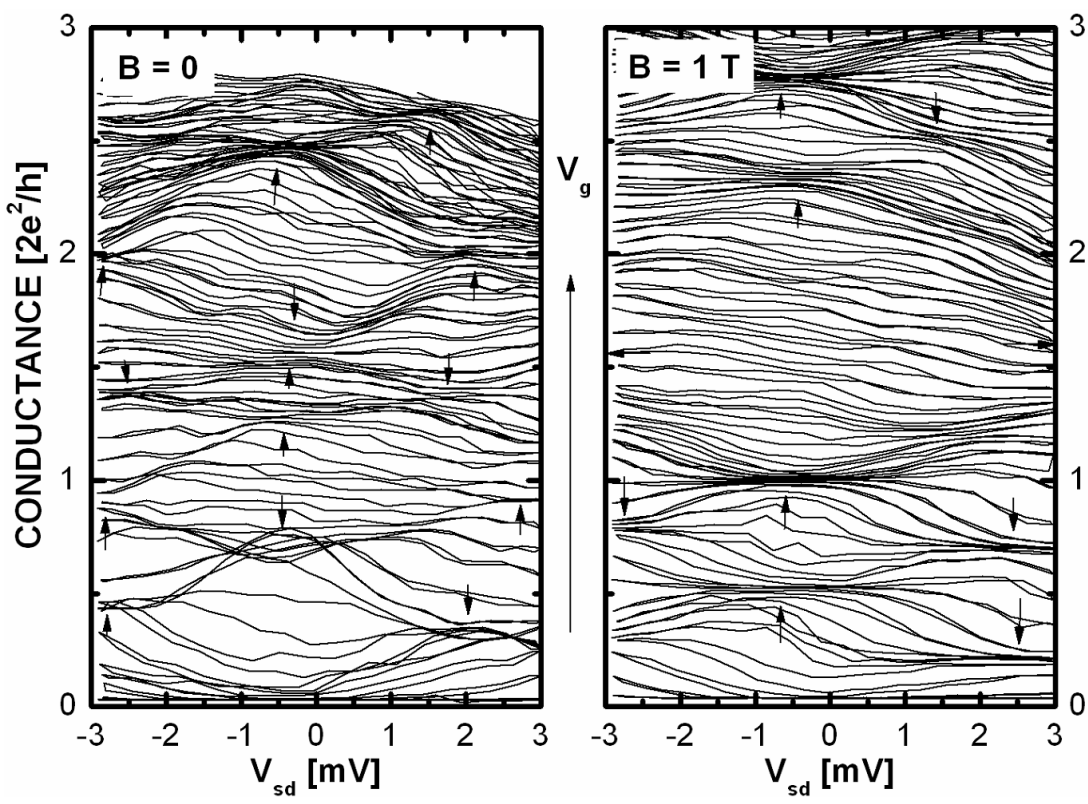

Fig. 8. Traces of differential conductance measured on PbTe wire vs. the applied source-drain voltage $V_{\mathrm{sd}}$, taken during a slow increase in the gate voltage, by $+4.6 \mathrm{mV}$ per each trace (from Ref. [14]). 
to the constriction and $\Delta E_{i j}$ is the energy difference between 1D quantum levels. Here, we present conductance measurements performed beyond this range, i.e. for $\mathrm{eV}>\Delta E_{i j}$. The result is presented in Fig. 8 for one constriction made of $25 \mathrm{~nm}$ thick $\mathrm{PbTe} \mathrm{QW}$. The gate geometry for this device is modified in comparison with that described in Sect. 2, in the way described in [14]. The nonlinear differential conductance is plotted as a function of the dc source-drain bias voltage, $V_{\mathrm{sd}}$. The gate voltage increases by $4.6 \mathrm{mV}$ by each $V_{\text {sd }}$ sweep. Conductance steps appear as an accumulation of traces. They are additionally indicated by arrows. Close to $V_{\text {sd }}=0$ we observe usual steps corresponding to the conductance quantization. In the absence of the magnetic field, their height is lowered with respect to the quantized values $n \times 2 e^{2} / h$, due to the slightly curved wire geometry. At large $V_{\text {sd }}$ values, we observe an additional "half-plateaux". This nonlinear effect appears when the number of conducting subbands for the two directions of transport differs by 1 [29]. From their positions, one can estimate difference between the ground and first excited $1 \mathrm{D}$ subbands, $\Delta E_{01} \approx 3 \mathrm{meV}$. If the magnetic field is applied, the quantization becomes accurate due to backscattering suppression, and a pronounced spin-resolved plateau of the bottom 1D level is observed. An estimated spin-splitting energy is close to the half of the Landau orbital splitting. For higher $V_{\mathrm{g}}$ we observe a complex behavior due to the contribution of $1 \mathrm{D}$ levels originating from excited 2D subbands of the starting PbTe quantum well. This is possible because the energy of the first excited subband in the $25 \mathrm{~nm}$ wide well is estimated to be only $6 \mathrm{meV}$.

\section{Summary}

The most important result of the reviewed studies is a demonstration of the conductance quantization in PbTe QWRs much more closely spaced from heavily doped regions than in any other system. Therefore, $\mathrm{PbTe}$ opens interesting possibilities for studying nanostructure physics. A uniqueness of this material relies on combination of excellent semiconducting properties, like a high electron mobility and tunable carrier concentration, with a paraelectric behavior leading to a huge dielectric constant at low temperatures. Because the nanoscale Coulomb potential fluctuations in $\mathrm{PbTe}$ are suppressed, one obtains a relatively easy access to the studies of the quantum ballistic phenomena. Our results prove that $\mathrm{PbTe}$ is a complementary candidate for fabrication of quantum devices. In particular, we have shown that the quantum level structure in $\mathrm{PbTe}$ nanostructures can be tuned by imposing $\mathrm{Pb}_{1-x} \mathrm{Eu}_{x}$ Te barriers. Therefore, this system allows for obtaining quantum ballistic nanostructures of different shapes and functionalities. Finally, we would like to emphasize that since the initial substrate layers used in this work have not yet been fully optimized (for example, the initial mobility was much lower than the records of bulk $\mathrm{PbTe}$ ), there is still much room for improvement of the devices. 


\section{Acknowledgments}

This work has been supported by the ERATO Semiconductor Spintronics project of the Japan Science and Technology Agency. The author would like to thank T. Dietl, J. Wróbel, M. Aleszkiewicz, E. Janik, E. Papis, E. Kamińska, A. Piotrowska, G. Springholz, and G. Bauer for essential contributions and helpful discussions.

\section{References}

[1] D. Loss, D.P. DiVincenzo, Phys. Rev. A 57, 120 (1998).

[2] R. Hanson, L.H. Willems van Beveren, I.T. Vink, J.M. Elzerman, W.J.M. Naber, F.H.L. Koppens, L.P. Kouwenhoven, L.M.K. Vandersypen, Phys. Rev. Lett. 94, $196802(2005)$.

[3] B.J. van Wees, H. van Houten, C.W.J. Beenakker, J.G. Williamson, L.P. Kouvenhoven, D. van der Marel, C.T. Foxon, Phys. Rev. Lett. 60, 848 (1988); D.A. Wharam, T.J. Thornton, R. Newbury, M. Pepper, H. Ahmed, J.E.F. Frost, D.G. Hasko, D.C. Peacock, D.A. Ritchie, G.A.C. Jones, J. Phys. C 21, L209 (1988).

[4] S. Tarucha, D.G. Austing, T. Honda, R.J. van der Hage, L.P. Kouwenhoven, Phys. Rev. Lett. 77, 3613 (1996).

[5] G.D.J. Smit, S. Rogge, J. Caro, T.M. Klapwijk, Phys. Rev. B 69, 035338 (2004).

[6] M.A. Topinka, B.J. LeRoy, R.M. Westervelt, S.E.J. Shaw, R. Fleischmann, E.J. Heller, K.D. Maranowski, A.C. Gossard, Nature (London) 410, 183 (2001).

[7] For review of PbTe properties, see e.g. J.I. Ravich, B.A. Efimova, I.A. Smirnov, Semiconducting Lead Compounds, Plenum, New York 1968; G. Nimtz, B. Schlicht, in: Springer Tracts in Modern Physics, Vol. 98, Ed. G. Höhler, Springer-Verlag, Berlin 1983, p. 1; Lead Chalcogenides Physics and Applications, Ed. D. Khokhlov, Taylor \& Francis, London 2003.

[8] See, e.g., G. Bauer, W. Jantsch, E. Bangert, in: Advances in Solid State Physics, Ed. P. Grosse, Vol. XXIII, Vieweg, Wiesbaden 1983, p. 27.

[9] G. Grabecki, J. Wróbel, T. Dietl, K. Byczuk, E. Papis, E. Kamińska, A. Piotrowska, G. Springholz, M. Pinczolits, G. Bauer, Phys. Rev. B 60, R5133 (1999).

[10] G. Grabecki, J. Wróbel, T. Dietl, E. Papis, E. Kamińska, A. Piotrowska, G. Springholz, G. Bauer, Physica E 13, 649 (2002).

[11] G. Grabecki, J. Wróbel, T. Dietl, E. Papis, E. Kamińska, A. Piotrowska, A. Ratuszna, G. Springholz, G. Bauer, Physica E 20, 236 (2004).

[12] G. Grabecki, J. Wróbel, T. Dietl, E. Janik, M. Aleszkiewicz, E. Papis, E. Kamińska, A. Piotrowska, G. Springholz, G. Bauer, Phys. Rev. B 72, 125332 (2005).

[13] G. Grabecki, J. Wróbel, T. Dietl, E. Janik, M. Aleszkiewicz, E. Papis, E. Kamińska, A. Piotrowska, G. Springholz, G. Bauer, Physica E 34, 560 (2006).

[14] G. Grabecki, J. Wróbel, P. Zagrajek, K. Fronc, M. Aleszkiewicz, T. Dietl, E. Papis, E. Kamińska, A. Piotrowska, G. Springholz, G. Bauer, Physica E 35, 332 (2006).

[15] G. Grabecki, J. Appl. Phys. 101, 081722 (2007). 
[16] W.D. Oliver, F. Yamaguchi, Y. Yamamoto, Phys. Rev. Lett. 88, 037901 (2002); D.S. Saraga, D. Loss, Phys. Rev. Lett. 90, 166803 (2003).

[17] M.J. Gilbert, J.P. Bird, Appl. Phys. Lett. 77, 1050 (2000).

[18] J. Wróbel, T. Dietl, A. Łusakowski, G. Grabecki, K. Fronc, R. Hey, K.H. Ploog, H. Shtrikman, Phys. Rev. Lett. 93, 246601 (2004); G. Grabecki, J. Wróbel, K. Fronc, M. Aleszkiewicz, M. Guziewicz, E. Papis, E. Kamińska, A. Piotrowska, H. Shtrikman, T. Dietl, Physica E 21, 451 (2004).

[19] G. Springholz, in: Lead Chalcogenides, Physics and Applications, Ed. D. Khokhlov, Taylor \& Francis, London 2003, p. 123.

[20] S. Yuan, N. Frank, G. Bauer, M. Kriechbaum, Phys. Rev. B 50, 5286 (1994).

[21] A. Prinz, G. Brunthaler, Y. Ueta, G. Springholz, G. Bauer, G. Grabecki, T. Dietl, Phys. Rev. B 59, 12983 (1999).

[22] G. Springholz, A.Y. Ueta, N. Frank, G. Bauer, Appl. Phys. Lett. 69, 2822 (1996).

[23] B. Tranta, H. Clemens, Lecture Notes in Physics, Vol. 301, Eds. G. Ferenczi, F. Beleznay, Springer-Verlag, Berlin 1988, p. 281.

[24] C.W.J. Beenakker, H. van Houten, in: Solid State Physics, Eds. H. Ehrenreich, D. Turnbull, Vol. 44, Academic, New York 1991, p. 1.

[25] C.W. Beenakker, H. van Houten, Phys. Rev. Lett. 63, 1857 (1989).

[26] B.L. Altshuler, B.I. Skhlovskii, Zh. Eksp. Teor. Fiz. 91, 220 (1986) [Sov. Phys. JETP 64, 127 (1986)]; P.A. Lee, A.D. Stone, H. Fukuyama, Phys. Rev. B 35, 1039 (1987).

[27] K.J. Thomas, J.T. Nicholls, M.Y. Simmons, M. Pepper, D.R. Mace, D.A. Ritchie, Phys. Rev. Lett. 77, 135 (1996).

[28] A.G. Scherbakov, E.N. Bogachek, U. Landman, Phys. Rev. B 53, 4054 (1996).

[29] N.K. Patel, J.T. Nicholls, L. Martin-Moreno, M. Pepper, J.E.F. Frost, D.A. Ritchie, G.A.C. Jones, Phys. Rev. B 44, 10973 (1991). 